\title{
Toward Construction of the Unified Lepton-Nucleus Interaction Model from a Few Hundred MeV to GeV Region
}

\author{
S. X. Nakamura*, Y. Hayato ${ }^{\dagger}$, M. Hirai**, H. Kamano ${ }^{\ddagger}$, S. Kumano ${ }^{\S}$, Il, M. Sakudall, \\ K. Saito** and T. Sato ${ }^{\dagger \dagger, \pi}$ \\ *Yukawa Institute for Theoretical Physics, Kyoto University, Kyoto 606-8542, Japan \\ ${ }^{\dagger}$ Kamioka Observatory, Institute for Cosmic Ray Research, University of Tokyo, Kamioka, Japan \\ ${ }^{* *}$ Department of Physics, Tokyo University of Science, Noda 278-8510, Japan \\ ${ }^{\sharp}$ Research Center for Nuclear Physics, Osaka University, Ibaraki 567-0047, Japan \\ ${ }^{\S}$ KEK Theory Center, Institute of Particle and Nuclear Studies, KEK, Tsukuba 305-0801, Japan \\ IIJ-PARC Branch, KEK Theory Center, Institute of Particle and Nuclear Studies, KEK, Tokai 319-1106, Japan \\ "Department of Physics, Okayama University, Okayama 700-8530, Japan \\ ${ }_{\dagger}^{\dagger}$ Department of Physics, Osaka University, Toyonaka, Osaka 560-0043, Japan
}

\begin{abstract}
Next generation neutrino oscillation experiments will need a quantitative understanding of neutrino-nucleus interaction far better than ever. Kinematics covered by the relevant neutrino-nucleus interaction spans wide region, from the quasi-elastic, through the resonance region, to the deeply inelastic scattering region. The neutrino-nucleus interaction in each region has quite different characteristics. Obviously, it is essential to combine different expertise to construct a unified model that covers all the kinematical region of the neutrino-nucleus interaction. Recently, several experimentalists and theorists got together to form a collaboration to tackle this problem. In this contribution, we report the collaboration's recent activity and a goal in near future.
\end{abstract}

Keywords: neutrino-nucleus interaction, neutrino oscillation

PACS: $13.15 .+\mathrm{g}$, 12.15.Ji, 25.30.Pt

\section{INTRODUCTION}

The breakthrough measurement of non-zero $\theta_{13}$ is changing the neutrino oscillation experiments into a new phase. The next target will be the leptonic CP violation, and the mass hierarchy of the neutrino. For aiming at this target, we need not only better controlled, higher statistics experiments but also a quantitative understanding of neutrino-nucleus interaction at the level of $5 \%$ or better. The relevant neutrino-nucleus interaction covers a wide kinematical region, as described in Fig. 1 (left). From the low to high energy side, characteristics of the neutrino-nucleus interaction changes: from the quasi-elastic (QE), through the resonance (RES) region, to the deeply inelastic scattering (DIS) region. Contribution from each reaction mechanism to the neutrino-nucleus interaction is displayed in Fig. 1 (right). There, $\mathrm{CC}$ (NC) stands for the charged-current (neutral-current). The single $\pi$ processes are associated with the resonance excitation. A long baseline accelerator neutrino experiment like T2K utilizes the neutrino-nucleus interaction at the left-bottom corner of Fig. 1 (left) where QE and the single pion production due to the $\Delta$ resonance excitation are main mechanism. Meanwhile, an atmospheric neutrino experiment expects to find effects of CP-violation/mass hierarchy in the higher resonance and DIS regions, as indicated in the figure. Obviously, it is essential to combine different expertise to construct a unified model covering all the kinematical region for the neutrino-nucleus interaction.

Recently, several experimentalists and theorists got together to form a collaboration at J-PARC branch of KEK Theory Center to tackle this problem [1]. Our strategy is to develop and combine baseline models for QE, RES and DIS. In this contribution, we report the current status of the baseline models for QE, RES and DIS, and discuss a future perspective.

\section{QUASI-ELASTIC REGION}

For the neutrino energy between $0.1 \mathrm{GeV}$ and $1 \mathrm{GeV}$, the $\mathrm{QE}$ process dominates the neutrino-nucleus interaction, as shown in Fig. 1 (right). At T2K energy, this QE process is the most dominant and important. Since the neutrino 

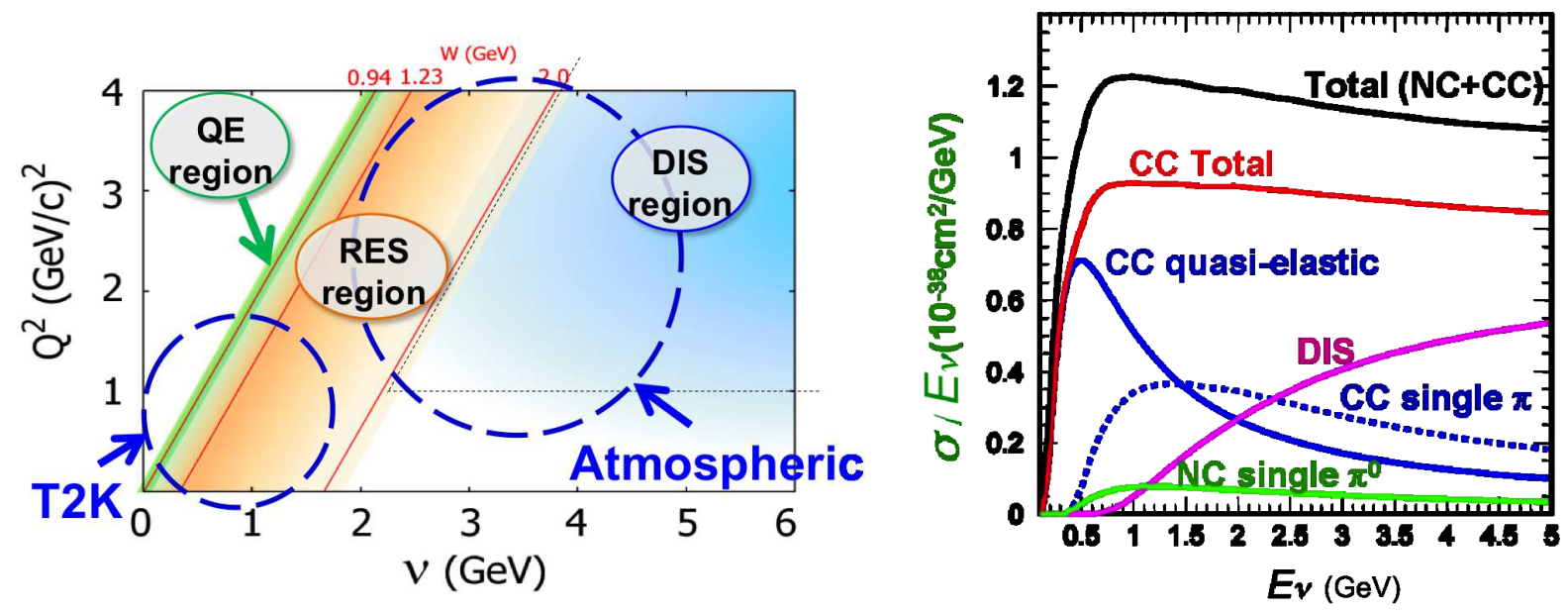

FIGURE 1. (Left) Kinematical region of neutrino-nucleus interaction relevant to the next-generation neutrino oscillation experiments. The neutrino energy and squared four-momentum transfer are denoted by $v$ and $Q^{2}$, respectively. (Right) Contribution of each reaction mechanism to the neutrino-nucleus interaction. The symbol $\sigma$ denotes the cross section.

oscillations depend on the energy of the neutrino, the neutrino oscillation experiments must reconstruct the neutrino energy by selecting events from the CC QE processes.

At the summary session of the NuInt12 Workshop, we generally agreed that the spectral function of nuclei should be used to calculate QE process and pion production in the neutrino-nucleus interactions, rather than a simple uniform nucleon momentum distribution based on the Fermi-Gas model, in order to achieve better accuracy of the calculations. The nuclear spectral functions $P(p, E)$ is the probability of removing a nucleon of momentum $(p)$ from ground state of the nucleus leaving the residual nucleus with excitation energy $(E)$. The nuclear spectral function naturally enables us not only to calculate both $\mathrm{CC}$ and $\mathrm{NC}$ QE cross section, but also to estimate a spectroscopic factor $\left(S_{p}\right)$ that is the probability of the residual nucleus in a specific shell state. Basic calculations with the spectral function approach are fully described in Ref.[2]. Comparisons of electron-nucleus scattering data with the spectral function approach and with the Fermi-Gas model are also shown in Ref.[2]. This approach is also useful to calculate CC and NC QE processes followed by a $\gamma$ emission [3] for which the branching fraction is significant $(\sim 40 \%)$.

A remaining issue is to better understand effects of $2 \mathrm{p}-2 \mathrm{~h}$ (2-particle-2-hole) configuration and/or MEC (mesonexchange current) in the QE process. This subject has been raised by several recent experiments on the QE cross section measurement [4]. The data seem to indicate a considerable contribution from $2 \mathrm{p}-2 \mathrm{~h}$ and/or MEC effects, and have motivated active theoretical studies [5].

\section{RESONANCE REGION}

The neutrino-nucleon interaction in the resonance region is a multi-channel reaction where not only single pion production but also two-pion production has a comparable contribution above the $\Delta(1232) . \eta$ and kaon productions can also happen with a smaller probability. In order to deal with this kind of multi-channel reaction, an ideal approach is to develop a unitary coupled-channels model. In the following subsections, we discuss the unitary dynamical coupled-channels (DCC) model, and its extension to forward neutrino-induced meson productions.

\section{Dynamical coupled-channels model}

In the DCC model $[6,7,8]$, we consider 8 channels: $\gamma N, \pi N, \eta N, \pi \Delta, \rho N, \sigma N, K \Lambda, K \Sigma$. We solve a coupled-channel Lippmann-Schwinger equation that contains meson-exchange potentials and bare $N^{*}$ excitation mechanisms, thereby obtaining unitary reaction amplitudes. We analyzed $\pi(\gamma) N \rightarrow \pi N, \eta N, K \Lambda, K \Sigma$ reactions data simultaneously up to $W=2.1 \mathrm{GeV}$ ( $W:$ total energy). The analysis includes fitting about 20,000 data points. The DCC model gives a 

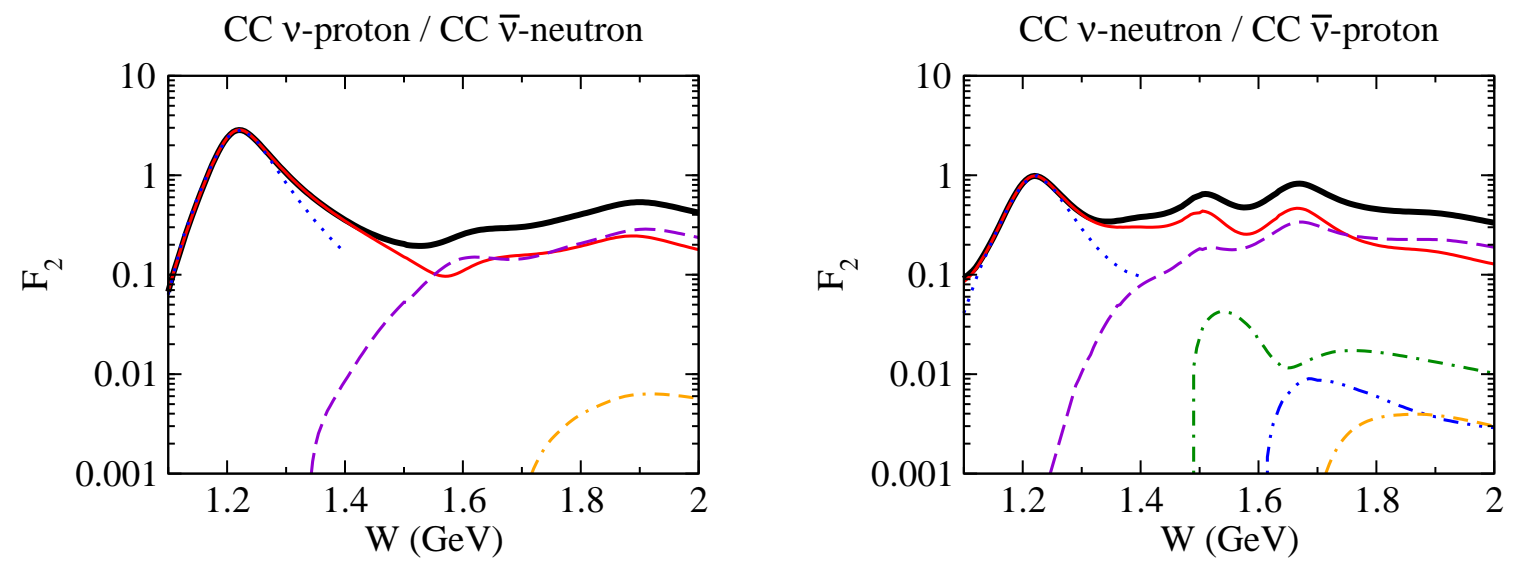

FIGURE 2. The structure function $F_{2}\left(Q^{2}=0\right)$ for the neutrino-induced meson productions from the DCC model. The solid (red), dashed (purple), dash-dotted (green), two-dotted dash (blue), and two-dash dotted (orange) curves are for the $\pi N, \pi \pi N, \eta N, K \Lambda$ and $K \Sigma$ reactions, respectively. The sum of them is given by the thick solid (black) curve.

reasonable description of meson production data in the resonance region, thus providing a good basis with which we proceed to the neutrino reactions.

\section{Forward neutrino-induced meson productions based on PCAC}

In the forward limit, the matrix element for a neutrino-induced meson production off the nucleon is given by the divergence of the axial current, accompanied by some kinematical factor. With the Partially Conserved Axial Current (PCAC) hypothesis, we can relate the matrix element with those of the pion-induced meson production. Thus with $\pi N \rightarrow X(X=\pi N, \pi \pi N, \eta N, K \Lambda, K \Sigma)$ amplitude, we can calculate the forward neutrino-induced meson productions. Recently, We have done such an application with the DCC model for $\pi N \rightarrow X$ [9]. We present the result in Fig. 2 where the structure function $F_{2}\left(Q^{2}=0\right)$ is shown as a function of the total energy $W$ of the hadronic final state. While $\pi N$ production is the dominant process up to $W=1.5 \mathrm{GeV}$, above that energy, the $\pi \pi N$ production becomes comparable to $\pi N$, showing the importance of the $\pi \pi N$ channel in the resonance region above $\Delta(1232)$. Other meson productions, $\eta N, K \Lambda$, and $K \Sigma$ reactions have much smaller contribution. We remark that this is the first prediction of the neutrino-induced $\pi \pi N, \eta N, K Y$ production rates based on a model that has been extensively tested by data.

\section{Future plan}

The current model for the neutrino reaction is limited to only the forward kinematics. In near future, we extend the model so that we can analyze the neutrino reaction of all kinematics in the resonance region. For that, we need to develop a dynamical model for both vector and axial currents. Regarding the vector current, we already have some part by having analyzed data for meson photoproductions on the proton. We will also analyze the neutron target data, so that we can decompose the vector current into isovector and isoscalar pieces; this decomposition is necessary for calculating the neutrino processes. The $Q^{2}$-dependence of the vector current couplings will be fixed by analyzing pion electroproduction data. For the axial current, we develop a model consisting of non-resonant mechanisms and also bare $N^{*}$ excitation mechanisms. The axial current couplings of $N-N^{*}$ transition can be determined from the corresponding $\pi N N^{*}$ coupling using the PCAC relation. The $Q^{2}$-dependence of the couplings can be fixed using available data of the neutrino-induced single pion production. In this way, we obtain all ingredients to calculate the neutrino-induced meson productions for finite $Q^{2}$ in the resonance region.

\section{DEEP INELASTIC SCATTERING REGION}

The deep inelastic region of neutrino-nucleon interactions corresponds to the kinematical conditions of $Q^{2} \gtrsim 1 \mathrm{GeV}^{2}$ and $W^{2} \gtrsim 4 \mathrm{GeV}^{2}$, and the cross sections or structure functions are described by the parton model. The neutrino- 
nucleon cross section via charged current is expressed by the three structure functions $F_{1}, F_{2}$, and $F_{3}$ :

$$
\left(\frac{d \sigma}{d x d y}\right)_{C C}^{v, \bar{v}}=\frac{G_{F}^{2} M_{N} E}{\pi\left(1+Q^{2} / M_{W}^{2}\right)^{2}}\left[F_{1}^{c c} x y^{2}+F_{2}^{c c}\left(1-y-\frac{M_{N} x y}{2 E}\right) \pm F_{3}^{c c} x y\left(1-\frac{y}{2}\right)\right],
$$

where \pm indicates $+(-)$ for $v(\bar{v}), x$ is the Bjorken scaling variable $x=Q^{2} /(2 p \cdot q)$ with the nucleon momentum $p$, the $W$-boson momentum $q$, and $Q^{2}=-q^{2}, M_{N}$ and $M_{W}$ are the nucleon and $W$-boson masses, $y$ is defined by $y=p \cdot q /(p \cdot k)$ where $k$ is the neutrino momentum, and $E$ is the neutrino-beam energy. In the leading twist and the leading order (LO) of the running coupling constant $\alpha_{s}$, the structure functions are expressed in terms of parton distribution functions (PDFs):

$$
\begin{aligned}
2 x F_{1}=F_{2}, \quad F_{2}^{v p}=2 x(d+s+\bar{u}+\bar{c}), \quad F_{2}^{\bar{v} p}=2 x(u+c+\bar{d}+\bar{s}), \\
x F_{3}^{v p}=2 x(d+s-\bar{u}-\bar{c}), \quad x F_{3}^{\bar{v} p}=2 x(u+c-\bar{d}-\bar{s}) .
\end{aligned}
$$

The next-to-leading order (NLO) expressions are calculated by the convolution integral of the above PDFs with NLO coefficient functions. For describing neutrino-nucleus cross sections, the nucleonic structure functions $F_{1,2,3}$ should be replaced by the corresponding nuclear ones $F_{1,2,3}^{A}$, which include nuclear corrections. The nuclear structure functions are expressed by nuclear PDFs (NPDFs) $f_{i}^{A}(x)$ for the parton type $i$ in the same way as Eq. (2). They contain nuclear correction factors $w_{i}(x, A, Z)$, which indicate deviations from a simple addition of proton $(p)$ and neutron $(n)$ contributions:

$$
f_{i}^{A}\left(x, Q_{0}^{2}\right)=w_{i}(x, A, Z) \frac{1}{A}\left[Z f_{i}^{p}\left(x, Q_{0}^{2}\right)+(A-Z) f_{i}^{n}\left(x, Q_{0}^{2}\right)\right],
$$

where $Z$ is the atomic number of a nucleus, $A$ is the mass number, and $Q_{0}^{2}$ is the initial $Q^{2}$ scale which is taken as $Q_{0}^{2}=1$ $\mathrm{GeV}^{2}$. The weight functions $w_{i}(x, A, Z)$ are determined by a global analysis of experimental data on lepton-nucleus deep inelastic scattering (DIS) and Drell-Yan processes with nuclear targets [10].

Neutrino DIS data can be included in the global analysis for extracting the NPDFs. However, one should be careful that nuclear modification data such as $F_{2}^{A} / F_{2}^{D}$, where $D$ indicates the deuteron, are not available in the neutrino scattering. Recently, there are some discussions on possible differences between nuclear modifications of chargedlepton and neutrino reactions. In the MINERvA project, structure functions of light nuclei will be measured, so that such an issue will be investigated independently.

In Fig. 1 (left), we notice a region which does not belong to either the resonance region or the DIS one at $Q \leq 1$ $\mathrm{GeV}^{2}$ and $W^{2} \geq 4 \mathrm{GeV}^{2}$. In order to provide a complete interaction model for various neutrino reactions, we need to describe such a region. At $Q^{2} \rightarrow 0$, there is a guiding principle like PCAC to connect a structure function to the pion scattering cross section. Together with the update of the NPDFs including new data, an appropriate description of this regions should be developed. These studies are currently in progress.

\section{ACKNOWLEDGMENTS}

The authors thank the J-PARC branch of the KEK theory center for supporting the collaboration's activity.

\section{REFERENCES}

1. http://j-parc-th.kek.jp/html/English/e-index.html

2. O. Benhar, N. Farina, H. Nakamura, M. Sakuda, and R. Seki, Phys. Rev. D 72, 053005 (2005).

3. A. Ankowski, O. Benhar, T. Mori, R. Yamaguchi, and M. Sakuda, Phys. Rev. Lett. 108, 052505 (2012).

4. R. Gran et al. (K2K), Phys. Rev. D 74, 052002 (2006); A. A.Aguilar-Arevalo et al. (MiniBooNE), Phys. Rev. Lett. 100, 032301 (2008); ibid Phys.Rev.D 81092005 (2010); V. Lyubushkin et al. (NOMAD), Eur. Phys. J. C 63355 (2009).

5. M. Martini, M. Ericson, G. Chanfray, and J. Marteau, Phys. Rev. C 80065501 (2009); Nieves, I. Ruiz Simo, and M.J. Vicente Vacas, Phys. Lett. B 70772 (2012).

6. A. Matsuyama, T. Sato, and T.-S. H. Lee, Phys. Rep. 439, 193 (2007).

7. H. Kamano, AIP Conf. Proc. 1374, 501-504 (2011).

8. H. Kamano, S. X. Nakamura, T.-S. H. Lee, and T. Sato, in preparation.

9. H. Kamano, S. X. Nakamura, T.-S. H. Lee, and T. Sato, Phys. Rev. D 86, 097503 (2012).

10. M. Hirai, S. Kumano, and M. Miyama, Phys. Rev. D 64, 034003 (2001); M. Hirai, S. Kumano, and T.-H. Nagai, Phys. Rev. C 70, 044905 (2004); Phys. Rev. C 76, 065207 (2007); M. Hirai, S. Kumano, and K. Saito, AIP Conf. Proc. 1189, 269 (2009). 